\title{
Tuberculosis of the Musculoskeletal System
}

\author{
David A. Spiegel, M.D.,* Girish K. Singh, M.D., $\dagger$ and Ashok K. Banskota, M.D.
}

\begin{abstract}
Summary: Tuberculosis remains a major source of morbidity and mortality worldwide, and orthopaedic surgeons working in developing nations, especially in South East Asia, are likely to encounter patients with osteoarticular manifestations of the disease. Chemotherapy is effective, and surgery serves as an adjunct for specific indications. Tuberculous osteomyelitis is the least common presentation, and the radiographic features may be confused with a variety of other diagnoses. A biopsy is required, and curettage may be performed in addition to chemotherapy. Bone Grafting is generally not required. The natural history of articular disease evolves over several years from a synovitis to joint destruction, and the prognosis is related to the stage of disease at presentation. In addition to chemotherapy, a synovectomy may be indicated in patients who have synovitis without significant joint destruction. For later stage disease, salvage options include osteotomy, arthrodesis, or prosthetic reconstruction. Approximately $50 \%$ of patients will have spinal involvement, and chemotherapy is effective in the majority of cases. The indications for surgery remain somewhat controversial, but may include uncertainty with the diagnosis, lack of response to chemotherapy, profound or progressive neurologic deficit, mechanical instability, or progression of deformity.
\end{abstract}

Tuberculosis has been a health concern for several thousand years, and millions continue to be afflicted with this disease. Although the disease may be found on all continents, orthopaedic surgeons working in south and Southeast Asia are most likely to encounter cases of osteoarticular tuberculosis. Tuberculosis is most common in areas with crowding, poor sanitation, and malnutrition. An increase in tubercular infection has been associated with the prevalence of the acquired autoimmune deficiency syndrome as well. While most cases are seen during the first 3 decades of life, the elderly popu-

From the Department of Orthopaedic Surgery, Children's Hospital of Philadelphia, Philadelphia, Pennsylvania, Department of Orthopaedic Surgery, The University Of Pennsylvania School of Medicine, and The Hospital and Rehabilitation Centre for Disabled Children, Banepa, Nepal; $†$ Department of Orthopaedics, King Georges Medical University, Lucknow, India, Department of Orthopaedics, BP Koirola Institute of Health Sciences, Dharan, Nepal; $\$$ Hospital and Rehabilitation Centre for Disabled Children, Banepa, Nepal, B\&B Hospital, Kathmandu, Nepal, Department of Orthopaedic Surgery, Kathmandu University School of Medicine.

Address correspondence and reprint requests to David A. Spiegel, M.D., Department of Orthopaedic Surgery, Children's Hospital of Philadelphia, $2^{\text {nd }}$ Floor Wood Building, $34^{\text {th }}$ Street and Civic Center Blvd., Philadelphia, PA 19104. E-mail: spiegeld@email.chop.edu lation is also susceptible. Only a small number of patients with tuberculosis will have osteoarticular involvement, half of which will have spinal disease. Although chemotherapy is highly effective, the most important variable predicting outcome is the stage of disease at presentation. Surgery is reserved for specific indications, most often to establish the diagnosis or to treat complications of the disease process. In addition, efforts should be made to improve the patient's general physiologic state. Our goal is to review the pathophysiology, clinical findings, diagnosis, and treatment options for osteoarticular tuberculosis.

\section{PATHOPHYSIOLOGY/NATURAL HISTORY}

The primary focus of disease is visceral (lungs, kidneys, lymph nodes), and musculoskeletal involvement occurs via hematogenous spread. ${ }^{70,71}$ Once deposited at a site, the organisms are ingested by mononuclear cells. Mononuclear cells then coalesce into epitheloid cells, and a tubercle is formed when lymphocytes form a ring around a group of epitheliod cells. Caseation then develops within the center of the tubercle. The host inflam- 
matory response intensifies, resulting in exudation and liquefaction, and a cold abscess is formed. A cold abscess is composed of serum, leukocytes, caseation, bone debris, and bacilli. The outcome depends on the characteristics and sensitivity of the organism, the status of the host immune system, the stage of disease at presentation, and the treatment. The range of end results may include resolution with minimal or no morbidity, healed disease with residual deformity, walled off lesions with calcification of caseous tissue, a low-grade chronic granular lesion, and local or miliary spread of the disease that may result in death. ${ }^{71}$

\section{CLINICAL FINDINGS}

Constitutional symptoms include low-grade fevers, night sweats, weight loss, anorexia, and malaise. Musculoskeletal complaints include swelling, stiffness, and pain ("night cries" may wake the patient from sleep). Osseous involvement is associated with localized warmth, swelling, and tenderness. Articular involvement is associated with tenderness, soft tissue swelling/effusion, and restriction of movement. Findings in patients with spinal disease include back pain/tenderness, a neurologic deficit, and a kyphotic deformity. Swelling and tenderness over a synovial bursa (especially the greater trochanter) or tendon sheath is seen less frequently. Lymphadenopathy is common, and sinuses are frequently observed (Fig. 1A,B).

\section{Management Principles}

The first priority is to establish the diagnosis. In regions of the world where tuberculosis is endemic, a therapeutic trial using the principle of treating rather than testing and treating is preferred when the typical clinical and radiographic features are present. There are neither the resources nor the manpower to subject every patient to a biopsy. If a biopsy is performed, the diagnostic yield is the greatest when material is submitted for smear, culture, and guinea pig inoculation. Cultures may take up to 8 weeks, and are only positive in $30 \%$ to $60 \%$ of cases. The likelihood of identifying the organism on a smear is $10 \%$ to $30 \%$. The Mantoux test will be positive in the majority of patients. Patients are often anemic, and the erythrocyte sedimentation rate is usually elevated. A chest radiograph should be ordered when the diagnosis is suspected or established. More advanced imaging studies may be unavailable, and in most cases are nondiagnostic.

Chemotherapy should be approximately $90 \%$ effective in eradicating the infection, provided that the appropriate agents are administered, and that patient compliance is ensured. Inadequate treatment and/or lack of compliance
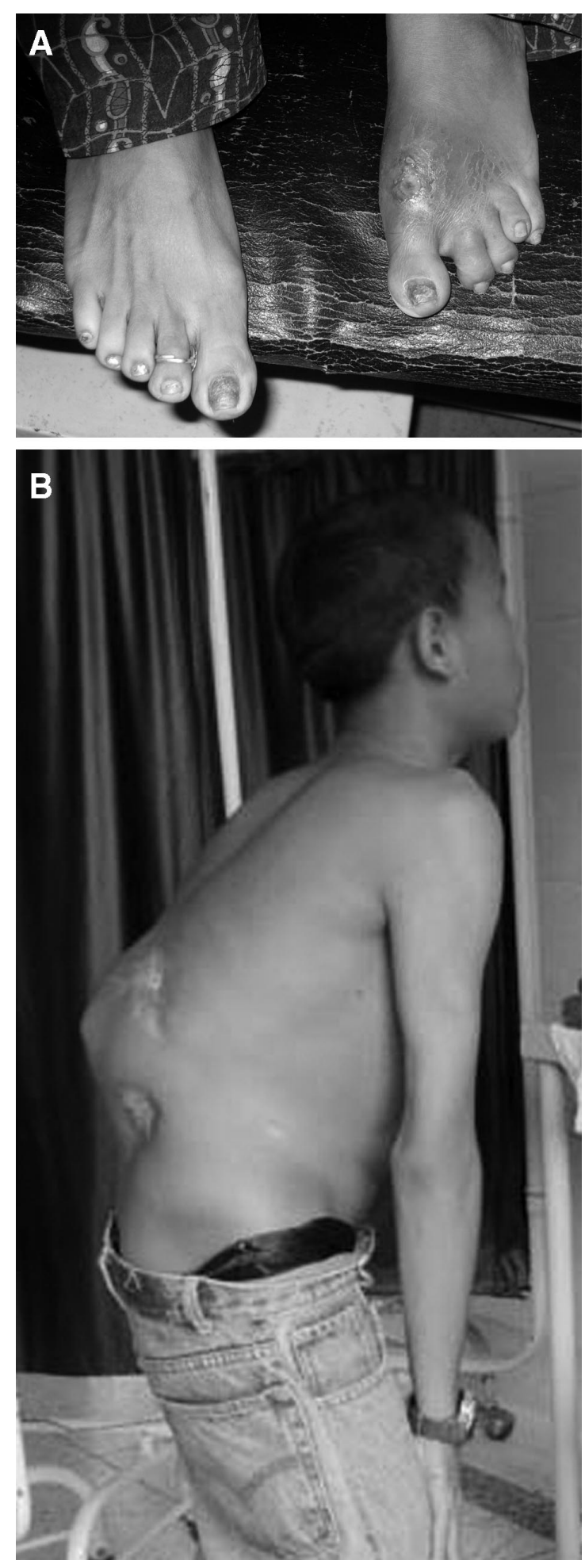

FIG. 1. (A,B) Sinuses are commonly observed with osteoarticular disease. An active sinus in the foot (A) demonstrates serosanguinous drainage. Sinuses usually heal with appropriate medical management, as shown in this patient with a significant kyphosis and evidence of multiple sinuses posteriorly. 
will result in the emergence of resistant organisms. The management of recurrence/relapse is challenging, expensive, and may fail to eradicate the disease. In the DOTS (directly observed treatment) program, compliance is ensured by employing local health care workers to document the ingestion of each dose of medication. ${ }^{5}$

Although treatment protocols vary, the current trend has been to use intermittent dosing (2-3 times per week), and to decrease the overall duration of therapy from 18 months to 9 months. $5,55,59,69$ Further study will be required to determine whether a shorter duration of therapy will be as effective for osteoarticular disease. Our recommended duration of therapy is usually 12 months, except for those with spinal disease, in whom we continue to recommend 18 months. Treatment is divided into two phases. During the first phase, 4 agents are administered for 2 to 3 months. In the second phase, 2 to 3 agents are prescribed over 4 to 6 months. Resistance to a single agent is in the range of $13 \%$, and multidrug resistance is encountered in approximately $1 \%$ to $2 \% .5,53$ The treatment of relapse typically involves 5 agents in the first phase, and 3 agents during the second phase. Failure to respond to standard chemotherapeutic protocols, with compliance documented, usually suggests the presence of a resistant organism or an alternate diagnosis.

Antitubercular drugs have been grouped into first and second line agents. 5,67,69 First line agents include Isoniazid, Rifampicin, Streptomycin, Pyrazinamide, Ethambutol, and Thiocetazone. Second line agents Capreomycin, Kanamycin, Ethionamide, Cycloserine, and Paraamino-salicylic acid. These agents will penetrate a tubercular abscess. ${ }^{76,77}$ Sinuses should heal within 6 to 12 weeks unless there has been a secondary bacterial infection. ${ }^{38}$

\section{Tuberculous Osteomyelitis}

Tuberculous osteomyelitis represents less than $5 \%$ of cases of osteoarticular tuberculosis. The duration of symptoms range from days to months, and coexisting visceral disease is uncommon. ${ }^{34,38}$ Abscess formation may occur, and sinuses are common. Notable features of a tuberculous sinus include bluish discoloration at the periphery, undermined edges, sero-sanguinous discharge, matted draining lymph nodes, and fixation to bone.

Radiographically, tuberculosis may be confused with a host of conditions (Fig. 2). The lesion may be metaphyseal or diaphyseal, and may penetrate the physis or extend into an adjacent joint. The most common presentation is a solitary lytic lesion, usually with a sclerotic rim. The differential diagnosis includes Brodie's abscess, chronic osteomyelitis, granulomatous lesions, and neoplasia. ${ }^{23,26,81}$ Sequestrae may be identified, ${ }^{38,71}$ and an
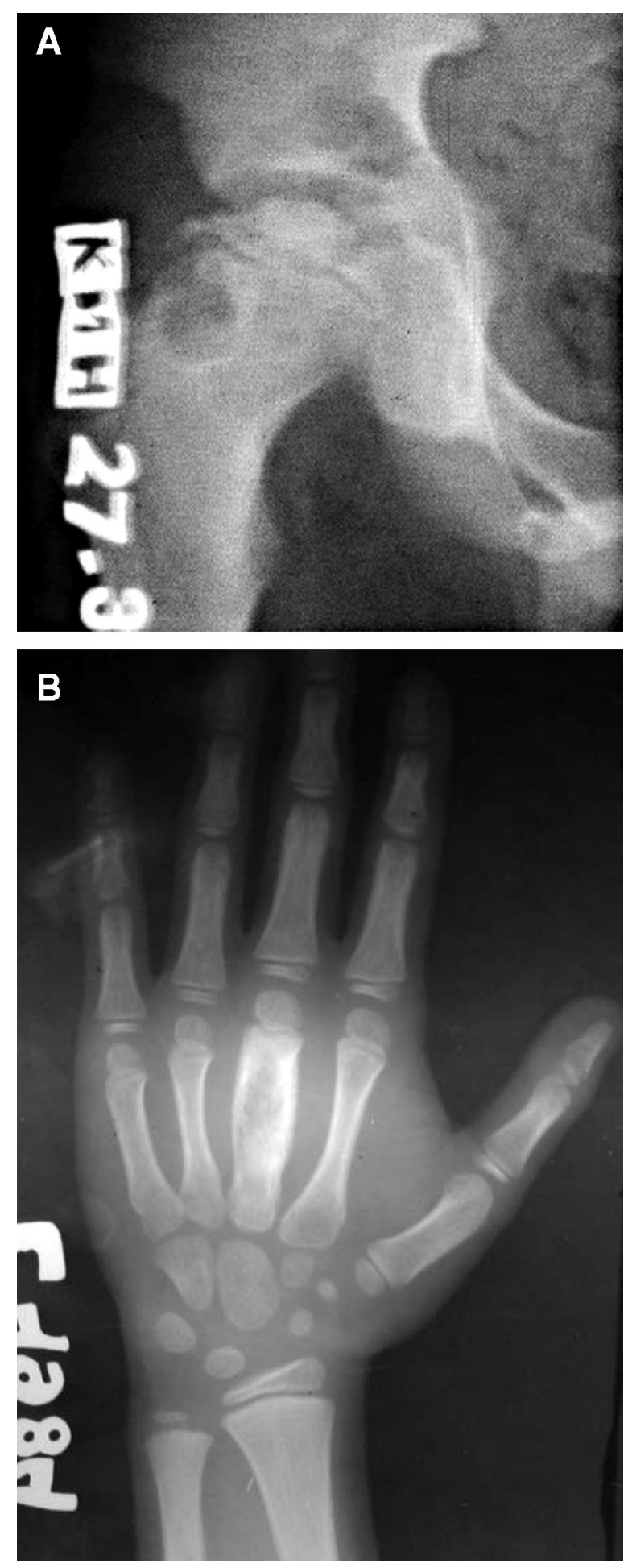

FIG. 2. (A-B) Tuberculous Osteomyelitis. The most common presentation is that of a lytic lesion with a sclerotic rim (A). Note the coexisting avascular necrosis. Spina ventosa $(\mathbf{B})$ represents a rare form of tuberculous osteomyelitis in which a spindle shaped expansion of the short tubular bones of the hand and foot is seen in association with multiple layers of subperiostial new bone. 
TABLE 1.

Tuli Classification. The Natural History of Tuberculous Arthritis Progresses Through 5 Stages [71]

\begin{tabular}{|c|c|c|c|c|}
\hline & Clinical Findings & Radiographic Findings & Treatment & $\begin{array}{l}\text { Anticipated } \\
\text { Outcome }\end{array}$ \\
\hline Stage I (Synovitis) & $\begin{array}{l}\text { 1.) Soft tissue swelling } \\
\text { 2.) } 75 \% \text { motion preserved }\end{array}$ & $\begin{array}{l}\text { 1.) Soft tissue Swelling } \\
\text { 2.) Osteopenia }\end{array}$ & $\begin{array}{l}\text { 1.) Chemotherapy } \\
\text { 2.) Rest } \\
\text { 3.) ROM } \\
\text { 4.) Splinting }\end{array}$ & $\begin{array}{l}\text { Normal or minimal } \\
\text { residua }\end{array}$ \\
\hline Stage II (early arthritis) & $\begin{array}{l}\text { 1.) Soft tissue Swelling } \\
\text { 2.) } 25-50 \% \text { loss of motion }\end{array}$ & $\begin{array}{l}\text { 1.) Soft tissue swelling } \\
\text { 2.) Marginal joint erosions } \\
\text { 3.) Diminution in joint space }\end{array}$ & $\begin{array}{l}\text { 1.) Chemotherapy } \\
\text { 2.) Rest } \\
\text { 3.) ROM } \\
\text { 4.) Splinting } \\
\text { 5.) Synovectomy }\end{array}$ & $50-70 \%$ mobility \\
\hline $\begin{array}{l}\text { Stage III (advanced } \\
\text { arthritis) }\end{array}$ & 1.) $75 \%$ loss of motion & $\begin{array}{l}\text { 1.) Marginal erosions } \\
\text { 2.) Cysts } \\
\text { 3.) Significant loss of } \\
\text { joint space }\end{array}$ & $\begin{array}{l}\text { 1.) Chemotherapy } \\
\text { 2.) Osteotomy } \\
\text { 3.) Arthrodesis } \\
\text { 4.) Arthroplasty }\end{array}$ & $\begin{array}{r}\text { Stable, painless joint } \\
\text { after salvage, with } \\
\text { or without motion }\end{array}$ \\
\hline $\begin{array}{l}\text { Stage IV (advanced } \\
\text { arthritis) }\end{array}$ & $\begin{array}{l}\text { 1.) } 75 \% \text { loss of motion } \\
\text { 2.) Subluxation or dislocation }\end{array}$ & 1.) Joint destruction & $\begin{array}{l}\text { 1.) Chemotherapy } \\
\text { 2.) Osteotomy } \\
\text { 3.) Arthrodesis } \\
\text { 4.) Arthroplasty }\end{array}$ & $\begin{array}{l}\text { Stable, painless joint } \\
\text { after salvage }\end{array}$ \\
\hline Stage V (Ankylosis) & 1.) Ankylosis & 1.) Ankylosis & $\begin{array}{l}\text { 1.) Chemotherapy } \\
\text { 2.) Osteotomy } \\
\text { 3.) Arthrodesis } \\
\text { 4.) Arthroplasty }\end{array}$ & Stable, painless joint \\
\hline
\end{tabular}

aggressive periostial response may be observed. ${ }^{23,38}$ In children, intraosseous thrombosis may result in sequestration of the diaphysis, which may easily be confused with chronic bacterial osteomyelitis. ${ }^{71}$

Unusual forms of skeletal tuberculosis include multiple cystic tuberculosis (one or more large, oval areas of rarefaction, children), ${ }^{23,33,66}$ disseminated skeletal tuberculosis (multiple osseous and/or articular sites, compromised host), ${ }^{1,2,34,51}$ closed multiple diaphysitis (swelling in forearms and legs in compromised children), ${ }^{2}$ and tuberculous dactylitis (metacarpal or phalanx). Spina ventosa, a spindle shaped expansion with multiple layers of subperiostial new bone, occurs in the short tubular bones of the hands and feet (Fig. 2B).

If feasible, a biopsy is recommended to establish the diagnosis. The zone of inflammation is large, and diagnostic material is most likely to be found inside the granulomatous focus, or in the synovium adjacent to a cyst. ${ }^{79}$ Curettage of the lesion is recommended, however, bone grafting is not necessary. ${ }^{26,63,70,71,80}$

\section{Tuberculous Arthritis}

The disease usually begins with seeding of the synovium by bacilli, but may also result from direct penetration of a metaphyseal focus. An effusion develops, and the synovium hypertrophies. Synovial granulation tissue proliferates at the joint periphery, and leads to marginal erosions. This granulation tissue gradually spreads across the joint from peripheral to central, resulting in loss of articular cartilage, and further bony erosions. This progresses to joint destruction, which may be associated with subluxation or dislocation. Ultimately, the joint becomes ankylosed.

The classification suggested by Tuli (Table 1) correlates the clinical and radiographic findings with both the recommended treatment and the expected outcome in patients with articular disease. ${ }^{71}$ Chemotherapy is recommended for all patients with active disease. During the early stages of disease, the goal is to obtain or maintain a normal or near normal range of motion. Rest is important, and the patient should be nonweightbearing. Range of motion exercises (active and active-assisted) are started when symptoms allow. Splinting helps to prevent deformity, and traction or serial casting may be used to restore or improve motion and/or alignment before splinting or bracing. Weightbearing (in a splint) is allowed when adequate alignment and motion are achieved, and the disease has been effectively controlled by chemotherapy. For patients presenting in the later stages of disease, the goal is to hold (splint or cast) the joint in a functional position, as ankylosis is expected. For those joints where ankylosis would not be well tolerated (hip and elbow), excisional arthroplasty might be considered. Corrective periarticular osteotomy may be required to reposition those joints that are ankylosed in a suboptimal position. There is also a role for prosthetic reconstruction. ${ }^{4,11,31,32}$ Current evidence suggests that there should be a significant disease free interval (ideally 
10 years) in between the completion of treatment and prosthetic implantation. Prophylactic chemotherapy for several weeks to months may allow earlier implantation, and chemotherapy can often salvage a prosthetic joint when reactivation of infection has been observed. Unfortunately, the availability of this technology is limited in the regions where tuberculous joint destruction is most likely to be encountered.

\section{SITE SPECIFIC ARTICULAR INVOLVEMENT}

\section{Hip}

The infection may originate in the synovium, the proximal femur (epiphysis, metaphysis, femoral neck, or trochanteric apophysis), the acetabulum, or the gluteal/ iliopsoas bursae. ${ }^{38,71}$ Cold abscesses may be palpable in the femoral triangle, the ischiorectal fossa, or the thigh. Sinuses may occur in any of these locations.

The radiographic findings vary considerably, depending on the primary location and degree of involvement, as noted by Shanmugasundaram. ${ }^{65} \mathrm{~A}$ lesion in the acetabular roof ("wandering acetabulum") may result in subluxation, and clinically there will be limb shortening without positioning. True pathologic dislocation may occur as well, which will be associated with both limb shortening and positioning. Protrusio may be associated with lesions in the acetabular floor (Fig. 3A). Coxa magna may be confused with Perthes disease in pediatric patients. Significant joint space narrowing without an osseous focus ("atrophic") may be difficult to differentiate from rheumatoid arthritis (Fig. 3B). Destruction on both sides of the joint may result in irregularity of the femoral head and incongruity ("mortar and pestle").

The treatment principles for joint disease have been outlined previously. In the early stages, a biopsy may be indicated for diagnosis, or for a lack of response to chemotherapy. ${ }^{7}$ The role of synovectomy and joint debridement is more controversial. While some authors feel that chemotherapy alone is sufficient, ${ }^{7}$ others recommend surgery when significant synovitis is present, but before joint destruction. ${ }^{4,37,71,83}$ In the later stages, traction or osteotomy may help realign the extremity, and casting can secure this position until the joint fuses. An alternative for those desiring motion at the expense of stability is excisional arthroplasty, when restoration of motion may take precedence over painless stability to allow cross legged sitting and squatting. 37,70,71,74 For those with a painless ankylosis in a nonfunctional position, osteotomy may be used to realign the limb. Arthrodesis is also an excellent option for those with pain or instability that don't need squatting or cross-legged sitting.
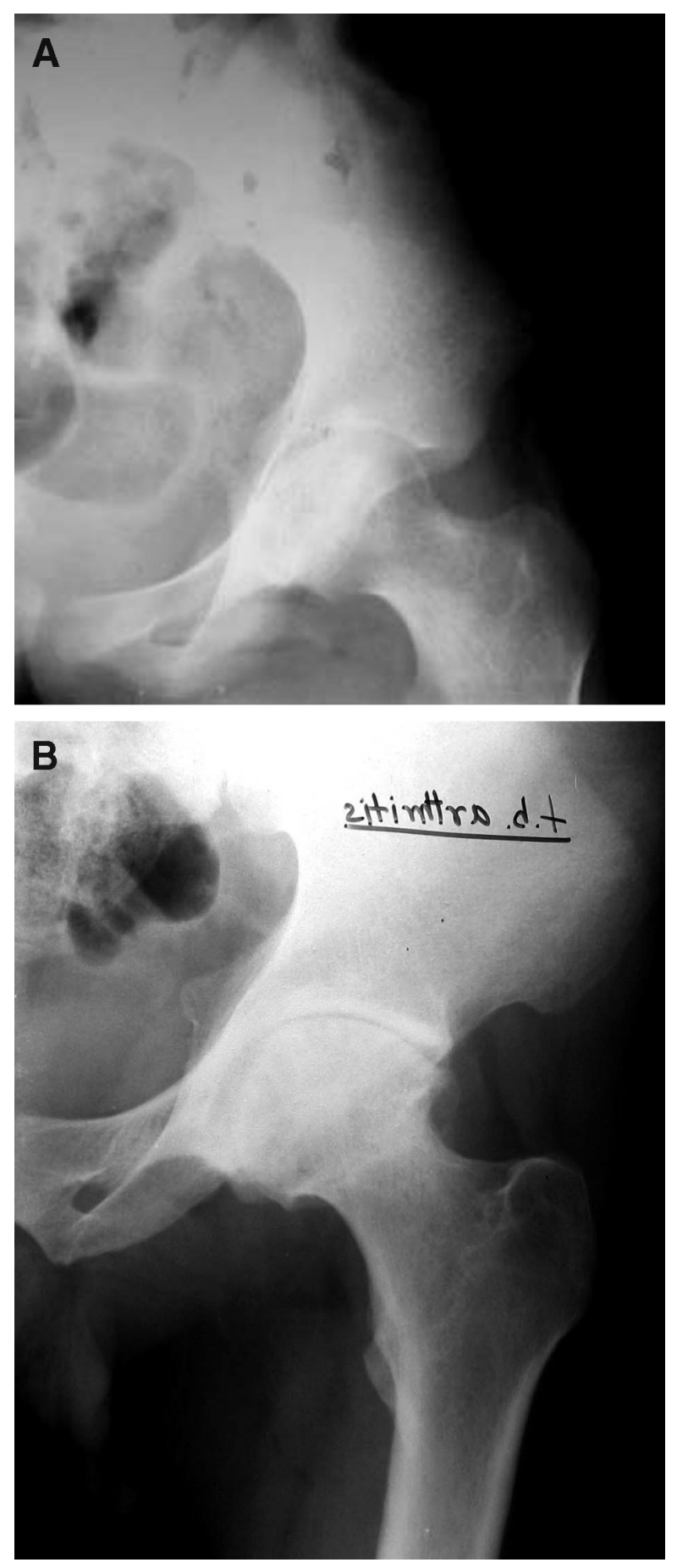

FIG. 3. Tuberculous of the Hip. Morphologic changes in the hip are diverse, and include a "protrusio" type (A) and an "atrophic" type (B).

\section{Other Joints}

The differential diagnosis for tuberculous arthritis of the knee includes rheumatoid arthritis, pigmented villonodular synovitis, hemophilia, and interarticular de- 

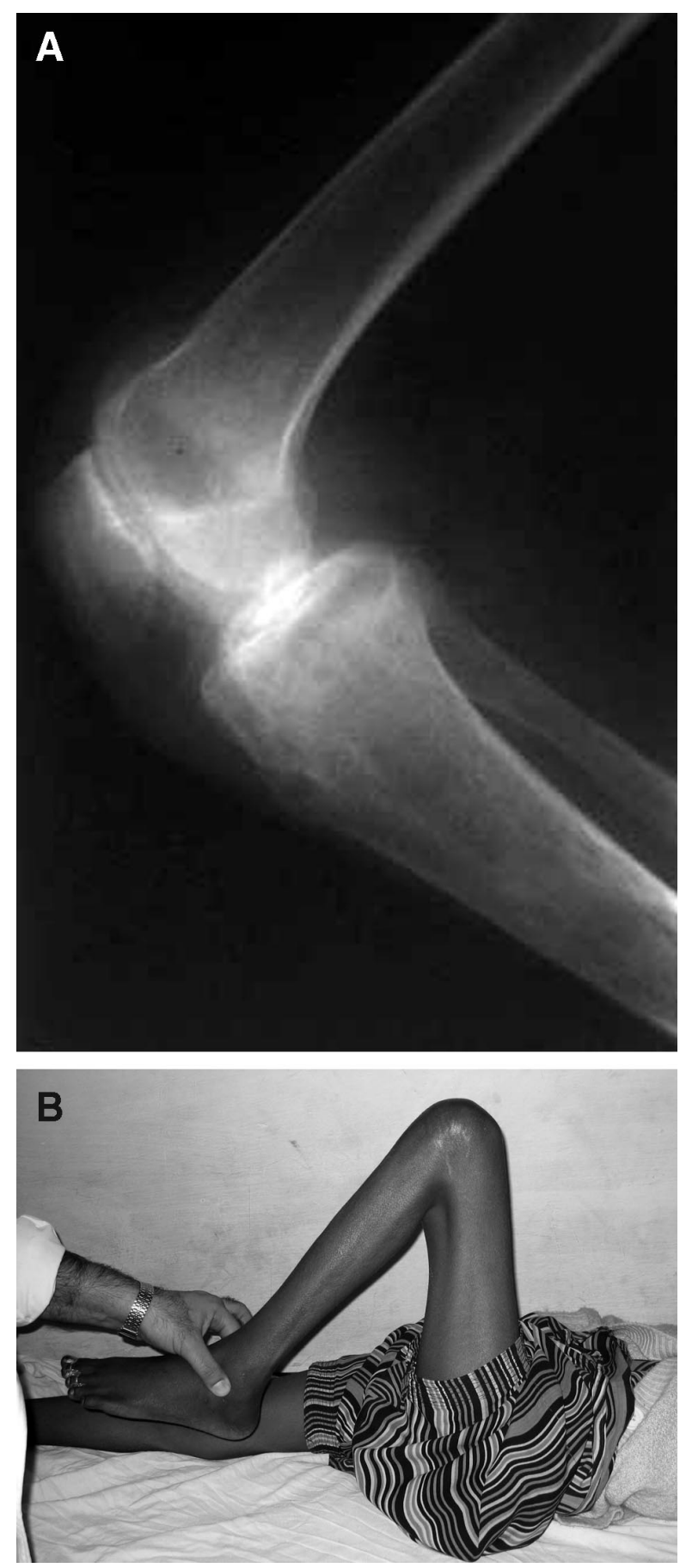

FIG. 4. (A-B) Tuberculous arthritis. In the early stages, there is regional osteopenia and soft tissue swelling, without any bony erosions or joint destruction (A). Another patient had a fixed flexion deformity of the knee $(\mathbf{B})$ at the time of presentation

rangement (Fig. 4A). Depending on the stage of disease, radiographic findings may include irregularity of the joint space, osteophytes, chondrocalcinosis, and loose bodies. ${ }^{9}$ A biopsy is often required to make the diagnosis, and synovectomy may be considered at the same time, although this remains controversial. $22,30,35$ During the early stages, traction and/or serial casting may be used to treat flexion contracture (with or without subluxation), and splinting in extension may help to prevent this complication (Fig. 4B).22,30 For who present at a late stage without deformity, immobilization is recommended until the joint fuses. Osteotomy may be required to reorient the joint, and surgical arthrodesis may be required if spontaneous fusion (or fibrous ankylosis) has not occurred. Prosthetic reconstruction is an option for a subset of patients, as mentioned previously. ${ }^{11,31}$

Tuberculosis of the foot and ankle occurs in less than $5 \%$ of cases. The calcaneus is most commonly affected, although any bone or joint may become involved. ${ }^{10,48,71}$ Local spread of disease may result in multiple sites of involvement. ${ }^{48,71}$ Cystic lesions with a well defined border an no sequestrae are most common. 48 "Rheumatoid" lesions have osteopenia associated with a diminution in joint space, and occur most frequently in the midfoot. Subperiostial scalloping may be seen on one or both ("kissing lesions") sides of the joint. Needle aspiration is an alternative to open biopsy, ${ }^{10}$ and surgical intervention may be indicated to arrest the local spread of disease. ${ }^{71}$ Surgery may be required to establish the diagnosis, to debride infected foci, and/or to stabilize joints.

Upper extremity involvement is uncommon. ${ }^{71}$ Disease in the shoulder usually occurs in adults, and is commonly a dry, atrophic form (sicca arthritis). The joint tends to become fixed in adduction, and arthrodesis may help to treat pain, stiffness, or instability. At the elbow, excisional arthroplasty may be preferable to arthrodesis for end stage disease. Involvement of the wrist is rare. Anterior dislocation of the carpus may be observed, and secondary involvement of the flexor tendon sheaths has been described. Splinting in dorsiflexion may be helpful, and occasionally arthrodesis is required.

\section{TUBERCULOUS SPONDYLITIS}

Approximately $50 \%$ of patients with osteoarticular tuberculosis will have spinal involvement. As modern chemotherapeutic protocols are highly effective in treating the disease, a major focus has been to prevent or treat kyphotic deformities.

Although the thoracic and thoracolumbar spine are involved most commonly, the disease may be seen in any region of the spine. Also, skipped lesions may occur rarely. The most common is presentation involves destruction adjacent to the endplates of two (or more) vertebral bodies (paradiscal type) (Fig. 5). The process 


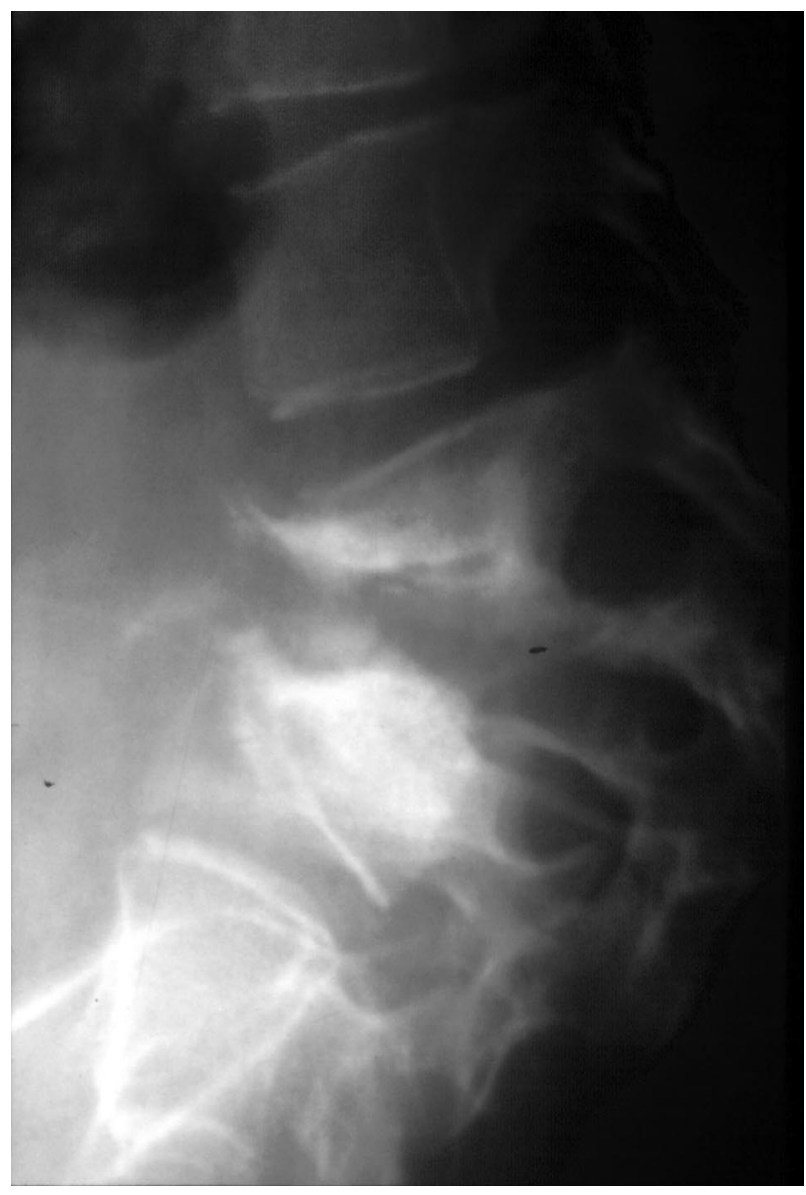

FIG. 5. Spinal Tuberculosis. This case involving the lumbar spine demonstrates involvement of three vertebrae anteriorly, and the bodies of L2 and L3 have collapsed and become fused.

begins with disc space narrowing, and the bony changes often do not appear for 3 to 5 months. Abscesses may track along the anterior and lateral margins, giving rise to vertebral scalloping ("aneurysmal phenomenon"). $\mathrm{Ab}$ scesses may be seen on both chest and spinal radiographs (Fig. 6). Abscesses below the diaphragm typically track along the psoas sheath, resulting in an asymmetry of the psoas shadow. Atypical radiographic findings include involvement of the posterior elements, circumferential involvement, lateral vertebral translation, involvement of a single vertebra or multiple vertebrae, and the spinal tumor syndrome. $3,54,58,68$

The differential diagnosis for spinal tuberculosis is large, especially in regions where the disease is less common. In addition to pyogenic vertebral osteomyelitis, other infections that may give a similar radiographic appearance include Salmonella typhi, Brucella, fungi (actinomysosis, blastomyscosis), and syphilis. Both benign (hemangioma, giant cell tumor, aneurysmal bone

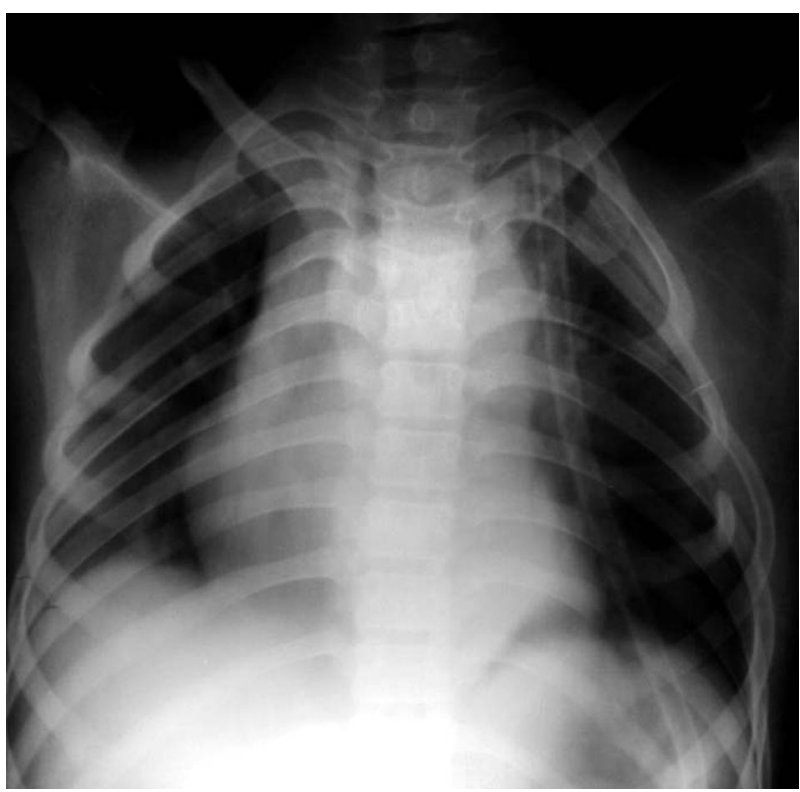

FIG. 6. Spinal Abscesses. Abscesses are commonly seen on the chest radiograph, appearing as an elongated density along the spine. The involved levels may be identified by a loss of disc space height, widening of the body, and collapse if present. Chronic abscesses of the spine or other locations may become calcified over time.

cyst, Histiocytosis X) and malignant tumors (Ewing's sarcoma, osteosarcoma, multiple myeloma, metastases) may also be included in the differential. Radiographic features that support the diagnosis of tuberculosis include multiple levels of involvement, relative sparing of the intervertebral disc, a large paravertebral abscess, subligamentous spread, multicentric involvement, and heterogeneous signal with rim enhancement on magnetic resonance imaging (MRI). ${ }^{14}$ Even under the best of circumstances, a tissue diagnosis is difficult to achieve. Chen et al. found that on biopsy, the smear was positive in only $15 \%$, and that histology was "typical" in $60 \%$ and "compatible" in $36 \% .^{8}$

Neurologic dysfunction is a dreaded complication of spinal tuberculosis. In general, the prognosis for recovery is good if the neurologic dysfunction develops gradually and is of short duration. A poor prognosis is encountered in patients with a complete paraplegia, flaccid paralysis, rapid development, longer duration of symptoms, and late onset of disease ("healed disease"). Spinal cord dysfunction may occur either during the "active" and "healed" phases of disease, and both the etiology and the prognosis differ considerably. The causes of neurologic compromise during the active phase are inflammatory edema, extradural compression from posterior extension of an abscess (pus, caseous material, granulation tissue, sequestrae), or an internal gibbus 


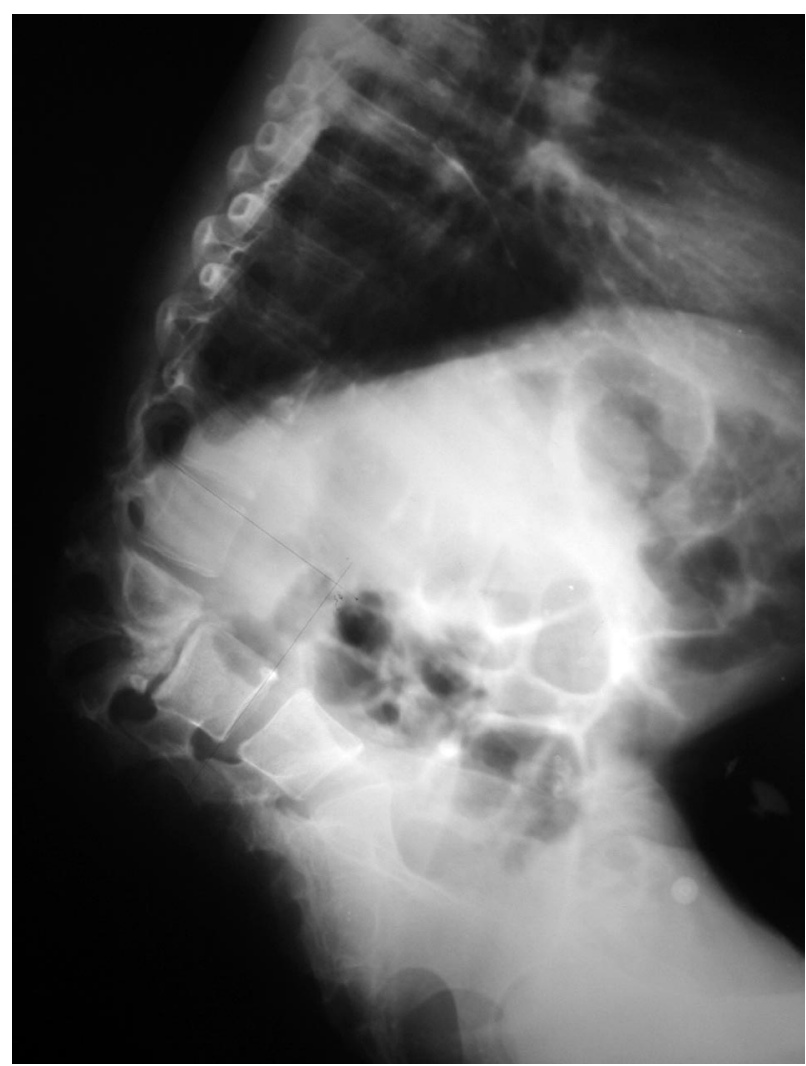

FIG. 7. An example of "healed" disease, with fusion of the L1 through L3 vertebral bodies. Although a significant kyphosis was observed in this teenager, he was asymptomatic and neurologically normal.

following collapse or malalignment of the involved vertebrae. ${ }^{20}$ Although the posterior longitudinal ligament usually protects the dura, direct invasion of the dura has been documented in a minority of cases. ${ }^{18,20}$ Rarely, there may be neurologic compromise without bony changes. A "spinal tumor syndrome" may result from tubercular granulomas in an extradural, intradural, or intramedullary location. Either an MRI or a myelogram will be necessary to make this diagnosis. Spinal cord infarction results in a sudden and irreversible paraplegia, and is fortunately rare. Neurologic compromise associated with "healed disease" (occurring more than 2 years after disease onset) may result from spinal stenosis, direct compression from an internal gibbus deformity, and constriction by peridural fibrosis (Fig. 7). ${ }^{20}$ With adequate treatment, the prognosis for neurologic recovery is generally good for patients with active disease, and guarded for those with healed disease. MRI findings associated with a poor prognosis include myelomalacia, thinning of the spinal cord, and the presence of a syrinx. ${ }^{28}$

Treatment begins with chemotherapy and general measures to improve the patient's physiologic state. Studies undertaken by the Medical Research Council of Great Britain found that outpatient chemotherapy is highly effective for "uncomplicated" spinal tuberculosis, and that the results were not improved by bedrest, by the use of a spinal orthosis, or by surgical debridement. ${ }^{39-47}$ If adequate resources were available, then surgical decompression and arthrodesis (Hong Kong procedure) resulted in faster healing and less deformity. It should be noted that exclusion criteria included patients with extraspinal disease, paraparesis of a degree that walking across a room was not possible, and recurrence of disease. Pattisson et al. studied 89 patients with neurologic deficits (active disease) treated by chemotherapy alone, and found that at latest follow up, $72 \%$ were neurologically normal, and $84 \%$ could ambulate with an assistive device. ${ }^{56}$ The duration of chemotherapy remains controversial for spinal disease. Traditionally, 18 months of chemotherapy has been recommended. Recently, Parthasarathy et al. have suggested that the duration of therapy may be reduced to 9 months. ${ }^{55}$

The role of surgery varies throughout different regions of the world, and both the indications for surgery and the specific procedures recommended remain somewhat controversial. To an extent, recommendations are based on the resources available locally, and the presence of surgeons with specialized training. The procedures are technically demanding, and intensive medical management is required during the perioperative period. The surgical treatment philosophy varies from routine decompression and arthrodesis (Hong Kong and others), to the "middle path" developed by Tuli in India, to chemotherapy alone when the resources for spinal surgery are unavailable. If Pattison's figures of $72 \%$ recovery with chemotherapy alone are to be generalized then surgery is needed in just $18 \%$ of these patients and criteria to identify them with sufficient post-test probability need to be established.

The "middle path" treatment approach reserves surgery for the treatment of complications of the disease. ${ }^{27,70-73,75}$ Patients are started on chemotherapy, and initially are managed with bedrest. Both the erythrocyte sedimentation rate and spinal radiographs are monitored at 3 to 6 month intervals. Patients are mobilized in a brace once their symptoms have subsided, and bracing is continued for a total of 18 to 24 months. Subcutaneous abscesses are aspirated, and $1 \mathrm{~g}$ of streptomycin may be instilled locally. Sinus tracts are excised if they remain after 12 weeks of chemotherapy. Surgical treatment is suggested for an increase in size of a paravertebral abscess despite adequate chemotherapy, involvement of the posterior elements, lack of clinical response after 3 to 
6 months of chemotherapy (neurologically normal), lack of neurologic recovery or progression of neurologic deficits after 3 to 4 weeks of chemotherapy, recurrence of disease, mechanical instability, or an uncertain diagnosis. Overall, surgery is recommended for approximately $5 \%$ of uncomplicated cases, and $60 \%$ of those with neurologic deficits.

According to Jain et al., the indications for surgery may include clinical factors (neural arch involvement, recurrent paraplegia, and massive retropharyngeal abscess causing difficulties with ventilation or swallowing), treatment factors (persistent or progressive deficit while following an adequate course of conservative treatment), imaging factors including panvertebral involvement [scoliosis or severe kyphosis on plain films, global destruction on compute tomography (CT) or MRI] or extradural compression (circumferential cord compression from granulation tissue on MRI), and patient factors (painful spasm or nerve root compression). ${ }^{28}$

While $80 \%$ of patients will have some localized kyphosis, only $3 \%$ to $5 \%$ progress to greater than $60^{\circ}$ (Fig. 8). ${ }^{59}$ Progression may occur during both the active phase and after healing..$^{59-61}$ Risk factors include age (children), thoracic involvement, multiple levels of involvement, and greater initial loss of vertebral height. Radiographic "at risk" factors include dislocation of the facets, retropulsion of diseased fragments, lateral translation of a vertebra, and toppling of a vertebra. ${ }^{61}$ Thus, prophylactic stabilization (with or without decompression) may be indicated for a subset of patients felt to be at high risk for the development of a significant kyphotic deformity. The treatment of deformity (and or paraplegia) associated with "healed disease" is challenging and fraught with complications. ${ }^{75}$ It should be noted that improvement in kyphosis may be observed in children, as the anterior vertebral growth centers may escape damage by the infection. ${ }^{61,62,64}$

As the pathology is usually anterior, the procedure most commonly used has been an anterior decompression and arthrodesis, as popularized in Hong Kong.6,18-21,25,29,36,78,82 A structural graft is essential to support the anterior column of the spine and to resist the progression of kyphosis. This approach may be technically difficult in the presence of severe kyphosis, in which case a lateral extrapleural approach may enhance visualization at the apex. Graft complications are more frequent when more than 2 disc spaces are involved, and include subsidence, resorption, fracture, and loss of position (Fig. 9).29,62 Progression of deformity may also be observed following a successful anterior arthrodesis, although the frequency and magnitude vary within the literature. Whereas Upadhyay et al. concluded that progression (posterior overgrowth) does not occur in chil-

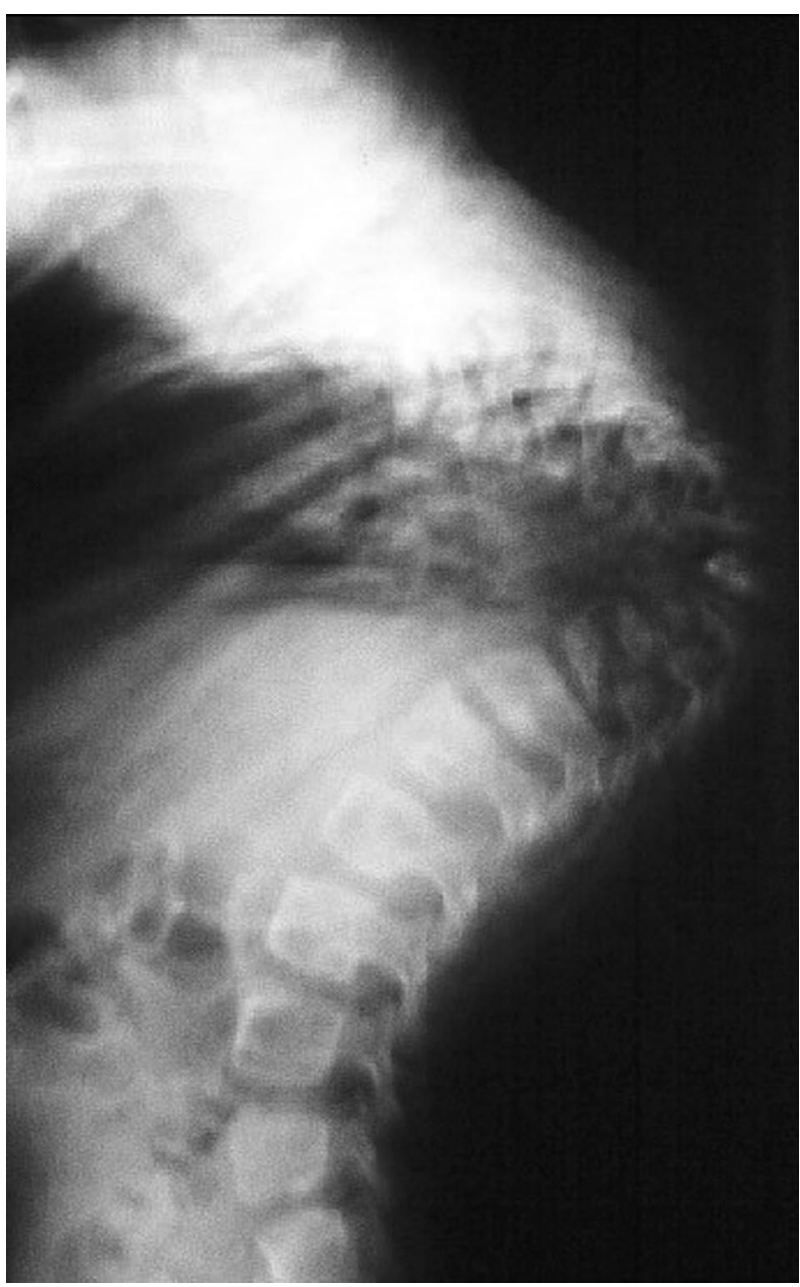

FIG. 8. A severe kyphosis develops in a minority of cases. This patient remained asymptomatic, as the kyphosis developed over several years. There remains a concern that he will develop neurologic dysfunction in the future. Risk factors include children with multiple levels of involvement in the thoracic spine, and a greater degree of vertebral collapse initially. Prophylactic stabilization of the spine should be considered in such high risk cases, if the resources are available.

dren, ${ }^{78}$ Rajasekaran observed an increase in kyphosis of more than $20^{\circ}$ in $22 \% .62$

Other approaches include a posterior spinal fusion, ${ }^{17}$ an anterior and posterior spinal fusion, a posterior spinal fusion followed by an anterior spinal fusion (same day or staged), costotransversectomy, and a lateral extrapleural approach. Both an anterior and posterior arthrodesis is recommended to treat the instability associated with circumferential disease. In addition, for those with greater than 3 levels of involvement, this approach may be the only method to reliably prevent the progression of kyphosis. Performing an instrumented posterior spinal fusion as the first stage, followed by a second stage anterior decompression and arthrodesis, may provide 


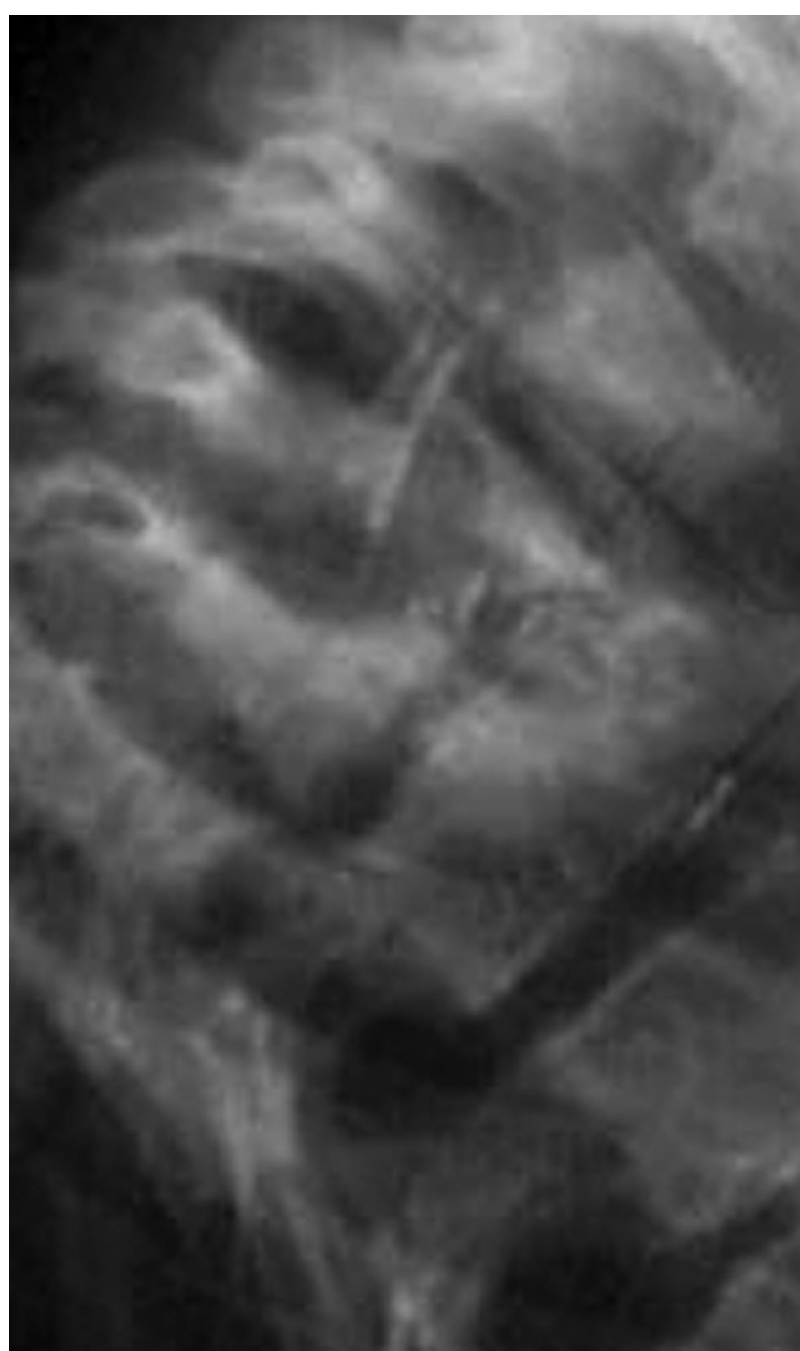

FIG. 9. Graft complications are frequent when more than 2 disc spaces are spanned. In this example, reactivation of disease (associated with noncompliance with chemotherapy) resulted in graft resorption, fracture, and persistent deformity.

protection for the anterior graft in situations where a longer segment must be spanned, and graft complications are likely. ${ }^{17,50,59}$ This approach is recommended when more than 3 levels are involved in the disease process. ${ }^{64,62}$ The posterior procedure should include 2 to 3 levels above and below the apex of the kyphosis. Costotransversectomy facilitates evacuation of a liquid abscess, but the exposure is insufficient for decompression and bone grafting. In this procedure, the medial portion of the rib and the underlying transverse process are removed, and an extrapleural dissection leads to the abscess. The lateral extrapleural approach is more versatile. Following removal of the medial portion of the rib and the transverse process, the pedicle is removed to allow greater access to the vertebral body and spinal cord. Typically, two to 3 levels must be exposed to facilitate debridement and bone grafting. The intercostal nerve is used as a guide to the foramen and spinal cord at each level to be exposed. A noninstrumented posterior spinal fusion may be performed through the same approach. ${ }^{13}$ Finally, a laminectomy is indicated in the rare case in which isolated involvement of the posterior elements is observed.

Sources for bone graft may include rib, iliac crest, and fibula. The limited information available suggests that allografts may also be used safely to support the anterior column of the spine. ${ }^{15,16}$ Instrumentation may be used in the presence of mycobacterial infection, ${ }^{52}$ and although posterior instrumentation has been used most frequently, the successful use of anterior implants has been reported as well. ${ }^{16,84}$

Cervical spinal involvement is uncommon, and patients typically present with pain, stiffness, and torticollis. ${ }^{12,24,27,36,71,72}$ Large abscesses may result in hoarseness, stridor, and dysphagia. Cervical lymphadenopathy, sinuses, and neurologic involvement are all commonly observed. Atlanto-axial involvement may result in instability at this articulation. Noncontiguous involvement may be seen, and involvement of more than two vertebrae is not uncommon in the mid-cervical spine. The lateral radiograph usually demonstrates widening of the retropharyngeal space in the presence of an abscess. In addition to chemotherapy, treatment recommendations have varied from the more conservative "middle path" protocol ${ }^{27,71,72}$ to routine decompression and arthrodesis. ${ }^{12,24,36} \mathrm{~A}$ uniform indication for surgery is when an abscess results in dysphagia, stridor, or difficulty with respiration. In the "middle path" approach, patients are started on chemotherapy and placed on bedrest with or without cervical traction, and an orthosis is occasionally recommended. The general indications for surgical decompression and arthrodesis have been outlined previously. Options for decompression include the transoral route, or an anterior approach along the anterior border of the sternomastoid muscle. Arthrodesis maybe accomplished either anteriorly or posteriorly.

The lumbar spine is also involved less frequently than the thoracic or thoracolumbar spine. ${ }^{49,57}$ Patients often present with pain, and neurologic dysfunction is uncommon. Kyphotic deformities are seen with some frequency. Chemotherapy is effective, and the indications for surgery remain unclear. While Moon et al. report a relatively benign course in 56 adults treated by chemotherapy alone, ${ }^{49}$ Pun et al. found late pain and degenerative changes in more than $50 \%$ of patients, with significant kyphosis in 14 of 26 patients. ${ }^{57}$ Compensatory lordosis in 
the upper lumbar and thoracic spine may be observed. Disease at the L5-S1 level is more difficult to address surgically, especially if grafting is deemed necessary.

\section{SUMMARY}

Osteoarticular tuberculosis may be seen with some frequency by health care professionals working in various regions of the world, particularly in South and Southeast Asia. Chemotherapy is extremely effective as long as the appropriate regimen is prescribed, and patient compliance is ensured. Surgical intervention is most commonly required to establish the diagnosis, and to treat the musculoskeletal complications of the disease, especially in cases with delayed presentation. Outcomes may be maximized through early detection and treatment, although adequate results can still be achieved with salvage procedures in patients presenting with late stages of disease.

\section{REFERENCES}

1. Aggarwal AN, Dhammi IK, Jain AK. Multifocal skeletal tuberculosis. Tropical Doctor 2001;31:219-220.

2. Babhulkar SS, Pande SK. Unusual manifestations of osteoarticular tuberculosis. Clin Orthop Rel Res 2002;398:114-120.

3. Babhulkar SS, Tayade WB, Babhulkar SK. Atypical spinal tuberculosis. J Bone Joint Surg 1984;66b:239-242.

4. Babhulkar SS, Pande S. Tuberculosis of the hip. Clin Orthop Rel Res 2002;398:93-99.

5. Bastian I, Colebnders R. Treatment and prevention of multidrugresistant tuberculosis. Drugs 1999;58:633-661.

6. Boachie-Adjei O, Squillante RG. Tuberculosis of the spine. Orthop Clin North Am 1996;27:95-103.

7. Campbell JAB, Hoffman EB. Tuberculosis of the hip in children. J Bone Joint Surg 1995;77b:319-326.

8. Chen WJ, Chen $\mathrm{CH}$, Shih $\mathrm{CH}$. Surgical treatment of tuberculous spondylitis. 50 patients followed for $2-8$ years. Acta Orthop Scand 1995;66:137-142.

9. Chow SP, Yau A. Tuberculosis of the knee-a long term followup. Int Orthop (SICOT) 1980;4:87-92.

10. Dhillon MS, Nagi ON. Tuberculosis of the foot and ankle. Clin Orthop Rel Res 2002;398:107-113.

11. Eskola A, Santavirta S, Konttinen YT, et al. Arthroplasty for old tuberculosis of the knee. J Bone Joint Surg 1988;70b:767-769.

12. Fang D, Leong JCY, Fang HSY. Tuberculosis of the upper cervical spine. J Bone Joint Surg 1983;65b:47-50.

13. Garst RJ. Tuberculosis of the spine: a review of 236 operated cases in an underdeveloped region from 1954-1964. J Spinal Disord 1992;5:286-300.

14. Griffith JF, Kumta SM, Leung PC, et al. Imaging of musculoskeletal tuberculosis: a new look at an old disease. Clin Orthop Rel Res 2002;398:32-39.

15. Govender S, Parbhoo AH. Support of the anterior column with allografts in tuberculosis of the spine. J Bone Joint Surg 1999;81b: $106-109$.

16. Govender S. The outcome of allografts and anterior instrumentation in spinal tuberculosis. Clin Orthop Rel Res 2002;398:60-66.

17. Guven O, Kumano K, Yalcin S, et al. A single stage posterior approach and rigid fixation for preventing kyphosis in the treatment of spinal tuberculosis. Spine 1994;19:1039-1043.
18. Hodgson AR, Stock FE. Anterior spine fusion for the treatment of tuberculosis of the spine. The operative findings and results of treatment in the first 100 cases. J Bone Joint Surg 1960;42a:295310.

19. Hodgson AR, Stock FE. Anterior spinal fusion. A preliminary communication on the radical treatment of Pott's disease and Pott's paraplegia. Br J Surg 1956;44:266-275.

20. Hodgson AR, Skinsnes OK, Leong CY. The pathogenesis of Pott's paraplegia. J Bone Joint Surg 1967;49a:1147-1156.

21. Hodgson AR, Stock FE, Fang HSY, et al. Anterior spinal fusion. The operative approach and pathological findings in 412 patients with Pott's disease of the spine. Br J Surg 1960;48:172-178.

22. Hoffman EB, Allin J, Campbell JAB, et al. Tuberculosis of the knee. Clin Orthop Rel Res 2002;398:100-106.

23. Hsieh CK, Miltner LJ, Chang CP. Tuberculosis of the shaft of the large long bones of the extremities. J Bone Joint Surg 1934;16a: $545-563$.

24. Hsu LCS, Leong JCY. Tuberculosis of the lower cervical spine (C2 to C7). A report on 40 cases. J Bone Joint Surg 1984;66b:1-5.

25. Hsu LCS, Cheng CC, Leong JCY. Pott's paraplegia of late onset. The causes of compression and results after anterior decompression. J Bone Joint Surg 1988;70B:534-538.

26. Huang $\mathrm{CH}$. Extra-articular tuberculous osteomyelitis. A report of 11 cases. Int Orthop (SICOT) 1996;20:169-171.

27. Jain AK, Kumar S, Tuli SM. Tuberculosis of spine (C1-D4) Spinal Cord 1999;37:362-369.

28. Jain AK. Treatment of tuberculosis of the spine with neurologic complications. Clin Orthop Rel Res 2002;398:75-84.

29. Jenkins DHR, Hodgson AR, Yau AMC, et al. Stabilization of the spine in the surgical treatment of severe spinal tuberculosis in children. Clin Orthop Rel Res 1975;110:69-80.

30. Kerri O, Martini M. Tuberculosis of the knee. Int Orthop 1985;9: 153-157.

31. Kim YH. Total knee arthroplasty for tuberculous arthritis. J Bone Joint Surg 1988;70a:1322-1330.

32. Kim YH, Han DY, Park BM. Total hip arthroplasty for tuberculous coxarthrosis. J Bone Joint Surg 1987;69a:718-727.

33. Komins C. Multiple cystic tuberculosis: a review and revised nomenclature, Br J Rad 1952;25:1-8.

34. Kumar K, Saxena MBL. Multifocal osteoarticular tuberculosis. Int Orthop (SICOT) 1988;12:135-138.

35. Lee AS, Campbell JAB, Hoffman EB. Tuberculosis of the knee in children. J Bone Joint Surg 1995;77b:313-318.

36. Lifeso R. Atlanto-axial tuberculosis in adults. J Bone Joint Surg 1987;69b:183-187.

37. Marmor L, Chan KP, Ho KC, et al. Surgical treatment of tuberculosis of the hip in children. Clin Orthop Rel Res 1969;67:133142.

38. Martini M, Adjrad A, Bouddjemaa A. Tuberculous osteomyelitis. A review of 125 cases. Int Orthop (SICOT) 1986;10:201-207.

39. Medical Research Council. A controlled trial of ambulant outpatient treatment and in-patient rest in bed in the management of tuberculosis of the spine in young Korean patients on standard chemotherapy. A study in Masan, Korea. J Bone Joint Surg 1973;55b:678-697.

40. Medical Research Council. A controlled trial of plaster-of-paris jackets in the management of ambulant outpatient treatment of tuberculosis of the spine in children on standard chemotherapy. A study in Pusan, Korea. Tubercle 1973;54:261-282.

41. Medical Research Council. A controlled trial of debridement and ambulatory treatment in the management of tuberculosis of the spine in patients on standard chemotherapy. A study in Bulawayo, Rhodesia. J Trop Med Hyg 1974;61:853-866.

42. Medical Research Council. A controlled trial of anterior spinal fusion and debridement in the surgical management of tuberculosis of the spine in patients on standard chemotherapy. A study in Hong Kong. Br J Surg 1974;61:853-866.

43. Medical Research Council. A five-year assessment of controlled trials of in-patient and out-patient treatment and of plaster-of-paris 
jackets for tuberculosis of the spine in children on standard chemotherapy. Studies in Masan and Pusan, Korea. J Bone Joint Surg 1976;58b:399-411.

44. Medical Research Council. Five-year assessments of controlled trials of ambulatory treatment, debridement and anterior spinal fusion in the management of tuberculosis of the spine. Studies in Bulawayo (Rhodesia) and in Hong Kong. J Bone Joint Surg 1978;60b:163-177.

45. Medical Research Council. A 10-year assessment of a controlled trial comparing debridement and anterior spinal fusion in the management of tuberculosis of the spine in patients on standard chemotherapy in Hong Kong. J Bone Joint Surg 1982;64b:393398.

46. Medical Research Council. A 10-year assessment of controlled trials of inpatient and outpatient treatment and of plaster-of paris jackets for tuberculosis of the spine in children on standard chemotherapy. Studies in Masan and Pusan, Korea. J Bone Joint Surg 1985;67b:103-110.

47. Medical Research Council. A 15-year assessment of controlled trials of the management of tuberculosis of the spine in Korea and Hong Kong. J Bone Joint Surg 1998;80b:456-462.

48. Mital R, Gupta V, Rastogi S. Tuberculosis of the foot. J Bone Joint Surg 1999;81b:997-1000.

49. Moon MS, Moon YW, Moon JL, et al. Conservative treatment of tuberculosis of the lumbar and lumbosacral spine. Clin Orthop Rel Res 2002;398:40-49.

50. Moon MS, Woo YK, Lee KS, et al. Posterior instrumentation and anterior interbody fusion for tuberculous kyphosis of dorsal and lumbar spines. Spine 1995;20:1910-1914.

51. O'Connor BT, Steel WM, Sanders R. Disseminated bone tuberculosis. J Bone Joint Surg 1970;52a:537-542.

52. Oga M, Arizono T, Takasita M, et al. Evaluation of the risk of instrumentation as a foreign body in spinal tuberculosis. Clinical and biologic study. Spine 1993;18:1890-1894.

53. Pablos-Mendez A, Raviglione MC, Laszlo A, et al. Global surveillance for antituberculosis-drug resistance, 1994-1997. N Engl J Med 1998;338:1641-1649.

54. Pande KC, Babhulkar SS. Atypical spinal tuberculosis. Clin Orthop Rel Res 2002;398:67-74.

55. Parthasarathy R, Sriram K, Santha T, et al. Short-course chemotherapy for tuberculosis of the spine. A comparison between ambulant treatment and radical surgery: ten year report. $J$ Bone Joint Surg 2001;81b:464-471.

56. Pattisson PRM. Pott's paraplegia: an account of the treatment in 89 consecutive patients. Paraplegia 1986;24:77-91.

57. Pun WK, Chow SP, Luk KDK, et al. Tuberculosis of the lumbosacral junction. J Bone Joint Surg 1990;72b:675-678.

58. Rahman NU. Atypical forms of spinal tuberculosis. J Bone Joint Surg 1980;62b:162-165.

59. Rajasekaran S. The problem of deformity in spinal tuberculosis. Clin Orthop Rel Res 2002;398:85-92.

60. Rajasekaran S, Shanmugasundaram TK. Prediction of the angle of gibbus deformity in tuberculosis of the spine. J Bone Joint Surg 1987;69a:503-509.

61. Rajasekaran S. The natural history of post-tubercular kyphosis in children: radiological signs which predict late increase in deformity. J Bone Joint Surg 2001;3b:954-962.

62. Rajasekram S, Soundarapandian S. Progression of kyphosis in tuberculosis of the spine treated by anterior arthrodesis. $J$ Bone Joint Surg 1989;71a:1314-1323.

63. Rasool MN, Govender S, Naidoo KS. Cystic tuberculosis of bone in children. J Bone Joint Surg 1994;76b:113-117.

64. Schulitz KP, Kothe R, Leong JCY, et al. Growth changes of a solidly fused kyphotic block after surgery for tuberculosis. Comparison of four procedures. Spine 1997;22:1150-1155

65. Shanmugasundaram TK. Bone and Joint Tuberculosis. Madras, India: Kothandaram and Co., 1983.

66. Shannon FB, Moore M, Houkom JA, et al. Multifocal cystic tuberculosis of bone. J Bone Joint Surg 1990;72a:1089-1092.

67. Shembekar A, Babhulkar S. Chemotherapy for osteoarticular tuberculosis. Clin Orthop Rel Res 2002;398:20-26.

68. Travlos J, Du Toit G. Spinal tuberculosis: beware the posterior elements. J Bone joint Surg 1990;72b:722-723.

69. Treatment of TB Guidelines for National Programs, 2nd ed. World Health Organization, Geneva, Switzerland, 1997.

70. Tuli SM. General principles of osteoarticular tuberculosis. Clin Orthop Rel Res 2002;398:11-19.

71. Tuli SM. Tuberculosis of the Skeletal System: Bones, Joints, Spine and Bursal Sheaths, 3rd ed. Bangalore: Jaypee Brothers, 2004.

72. Tuli SM. Tuberculosis of the craniovertebral region. Clin Orthop Rel Res 1974;104:209-212.

73. Tuli SM. Result of treatment of spinal tuberculosis by the "middle path" regime. J Bone Joint Surg 1975;56b:13-23.

74. Tuli SM, Mukherjee SK. Excision arthroplasty for tuberculosis and pyogenic arthritis of the hip. J Bone Joint Surg 1981;63b:29-32.

75. Tuli SM. Severe kyphotic deformity in tuberculosis of the spine: current concepts. Int Orthop 1995;19:327-331.

76. Tuli SM, Kumar K, Sen PC. Penetration of antitubercular drugs in clinical osteoarticular tubercular lesions. Acta Orthop Scand 1977 48:362-368.

77. Tuli SM, Mishra S. Penetration of antitubercular drugs in cold abscesses of skeletal tuberculosis and in tuberculous joint aspirates. Ind J Orthop 1983;17:14-18.

78. Upadhyay SS, Saji MJ, Sell P, et al. The effect of age on the change in deformity after radical resection and anterior arthrodesis for tuberculosis of the spine J Bone Joint Surg 1994;76A:701-708.

79. Versfeld GA, Solomon A. A diagnostic approach to tuberculosis of bones and joints. J Bone Joint Surg 1982;64b:446-449.

80. Vohra R, Kang HS, Dogra S, et al. Tuberculous osteomyelitis. J Bone Joint Surg 1997;79b:562-566.

81. Wang MNH, Chen WMC, et al. Tuberculous osteomyelitis in young children. J Ped Ortho 1999;19:151-155.

82. Watts HG, Lifeso RM. Tuberculosis of bones and joints: curren concepts review. J Bone Joint Surg 1996;78a:288-298.

83. Wilkinson MC. Tuberculosis of the hip and knee treated by chemotherapy, synovectomy and debridement: a follow-up study. J Bone Joint Surg 1969;51:1343-1359.

84. Yilmaz C, Selek H, Gurkan I, et al. Anterior instrumentation for the treatment of spinal tuberculosis. J Bone Joint Surg 1999;81a: 1261-1277. 